\title{
Mechanisms of Progress in Organic and Cultural Evolution
}

\author{
Börje Ekstig ${ }^{1}$ \\ ${ }^{1}$ Uppsala University, Department of Education, 75002 Uppsala, Sweden. \\ Correspondence: Börje Ekstig, Uppsala University, Department of Education, 75002 Uppsala, Sweden.
}

Received: December 7, 2015

Accepted: December 22, 2015

Available online: January 13, 2016

doi:10.11114/ijsss.v4i2.1301

URL: http://dx.doi.org/10.11114/ijsss.v4i2.1301

\begin{abstract}
Due to the randomly changing environmental conditions in which natural selection is acting, it is hard to see how this process could give evolution the steady direction of a large-scale incessant progress. In the present article I propose a solution to this dilemma by the application of the concept of complexity as a measure of evolutionary progress, a notion that I have developed in a descriptive way in previous works. By means of the concept of complexity one can conceive of the evolutionary process as having a stable direction towards ever-increasing complexity. The explanatory mechanism behind this trend is in the present work suggested to be found in the combined actions of natural selection, competition, feedback, arms race, and sexual selection. These mechanisms are discussed at length, being applicable not only in organic evolution but in human cultural and social evolution as well.
\end{abstract}

Keywords: Evolution, Culture, Complexity, Competition, Feedback, Language acquisition, Sexual selection, Arms race.

\section{Introduction}

As is well known, Charles Darwin's theory of evolution implies that organisms evolve by a continuous adaptation to their environment by means of natural selection. There are few biologists today - and I'm certainly not one - who would venture to attack this solid structure. However, during the long time of the evolution of life, the environment has certainly changed dramatically in a highly random way and under such chaotic conditions it is hard to see how natural selection could have driven life to evolve the way it actually has, characterized as it is by a large-scale incessant progress. It is my intention with this article to tackle this problem. In this enterprise I will try to find a solution that might be applicable to the human cultural and social evolution as well.

As soon as there are several species (or cultures) living and thrivling simultaneously in contact with one other, there is competition. This mechanism lies at the heart of the very process of life and can be seen as a form of natural selection if one includes the actions of other individuals of the same species or those of other species as parts of the external environment. Indeed, it can be characterized by the popular expression "the struggle of life". This struggle in many cases compels creatures to change, if possible, towards increased complexity. Therefore competition is intimately connected to the process of evolution.

Another fundamental characteristic of the processes of biological as well as of cultural evolution is that it proceeds in an iterative process. Every new generation starts so to say from scratch, just retaining and reusing the genetic and cultural information transmitted from the preceding generation. This process gives the individual developmental growth an especially central role in the evolutionary process.

The importance of the developmental process is furthermore strengthened by the fact that natural selection primarily acts on the developmental process, not on the evolutionary process directly. This is so because evolution proceeds by means of changes in genes, which have direct impact on the developmental process only. Thus, evolution advances as a result of insensibly small continuous modifications in the development programs of living organisms; modifications giving rise to the vast diversity of life. A corresponding rationale can be made about cultural evolution and I maintain that much of our understanding of cultural and social evolution has to be based on an understanding of children's mental, verbal and social development.

The transmission of genetic and cultural information from each generation to the beginning of the next can be considered as a feedback process. I have in a previous publication (Ekstig, 2016) made a first analysis of the concept of feedback, an analysis that I expand in the present work, especially to the field of human cultural and social evolution. 
Life and human culture involve processes of extremely high complexity. Charles Lineweaver and coauthors have collected contributions to the analysis of complexity from several researchers in the field. These authors point out a central problem with the concept of complexity inasmuch as there is no definition generally agreed on. But, as the editors ask, even without a definition or a way to measure it, isn't it qualitatively obvious that biological complexity has increased? Do we really need to wait for a precise definition to think about complexity and its limits? (Lineweaver et al., 2013 p. 5).

I have in preceding publications (Ekstig, $2010 \mathrm{~A}, 2015)$ suggested the concept of complexity to be useful in the analysis of the evolutionary process, both regarding its organic and cultural manifestations. In particular, I have demonstrated complexity to increase continuously from the realm of organismal evolution into the human cultural evolution thus fusing these two processes, so different if superficially regarded, into a large-scale unified process. I have found that the concept of complexity allows us to conceive of the evolutionary process as having a well-defined direction towards ever-increasing complexity. In addition, I have shown that an environment-independent form of natural selection is to be included in the explication of the trend of increasing complexity (Ekstig, 2016). In the present work, I discuss additional explications to be found in the combined actions of ordinary natural selection, competition, feedback and sexual selection.

\section{Natural selection and cultural evolution}

A challenging question is to what extent the Darwinian theory of biological evolution could serve as a unifying principle, thus also including the human cultural progression. Edward O. Wilson, in developing the theory of sociobiology, claims that gene-culture coevolution is a special extension of the more general process of evolution by natural selection (Wilson, 1998 p. 128). Wilson emphasizes that culture and hence the unique qualities of the human species will make complete sense only when linked in causal explanation to the natural sciences, biology in particular (ibid., p. 267).

Wilson's ideas have turned out to be highly controversial but got their follow-up by Richard Dawkins (1976) with his introduction of the concept of memes, forming a corresponding kind of hereditary unit in the human cultural evolution as that of genes in biological evolution. Dawkins ideas have been expanded by Daniel Dennett (1995), suggesting that the Darwinian process, involving variation, selection and retention, may be seen as a substrate-neutral evolutionary algorithm that could be applied to many fields of knowledge outside that of biology. He notes in particular the importance of memetics in social science.

Likewise, Susan Blackmore (1999) discusses the applicability of the notion of memes to the evolution of human culture in which she accentuates the importance of imitation. She notes that human brains began expanding in size at about the same time that we started using stone tools and suggests that once individuals began to imitate each other, selection pressure favoured those who could make the most intelligent choices on what to imitate. Imitation led to the emergence of language, in the progression of which sexual selection plays a crucial role (ibid., pp. 69 - 77).

\section{Competition}

In considering the fact that external contingencies certainly have been highly chaotic during the long existence of living creatures on earth, its steady direction of increasing of complexity cannot be explained merely by Natural selection as working by means of adaptations to these unstable environmental conditions. I suggest that competition might in part explain the steady increase of complexity.

Competition is a general source of evolutionary change and is by no means a new concept. Living creatures, in order to survive, continuously have to compete about food, leading to ordinary Darwinian natural selection, about mates, leading to sexual selection, and about ecological room in the external environmental landscape. Furthermore, there is competition of ideas that can survive and be dispersed in human brains and social life. What is new in my analysis and application of competition is that it can be seen as a process in the complexity space thus implying a powerful principle in the study of the large-scale characteristics of evolution.

It is commonly observed that most species don't change much over long periods of their evolutionary history. They apply what is called stabilizing selection. I suggest this feature to be explained as a consequence of the fact that the complexity space is tightly occupied by other species above as well as below the specific level of each species, in this way acting as competitors. Daniel Dennett expresses this view, in stating that the odds are heavily against any mutation being more viable than the theme on which it is a variation (Dennett, 1995 p. 89). It is only the species that at each point of time in the evolutionary history dwells on the highest level of complexity that may evolve to a still higher level because for those, there are no competing species at higher levels. These species may then apply what is called disruptive selection. This process explains much of the emergence of new species and gives the evolutionary process a recognizable direction in a steady though stepwise increasing complexity. It should be remembered, though, that this 
conclusion is depending on an acceptance of the concept of complexity as a quantitative concept.

The reasoning fulfils my intention as expressed in the introduction to find an explanation to the large-scale incessant progress of evolution going on in spite of the randomly changing environment. A main characteristic of this process is that it is cumulative. Actually, it seems that a species, despite hard conditions, will never regress to a former stage, except maybe parasites. In hard conditions they will rather choose extinction. This is clearly expressed by Harold Blum although without the application of the concept of complexity: "The whole picture of evolution is one in which derivation of new patterns comes from modification of those existing - never by the return to a former starting place for a new "try" (Blum, 1968 p.175).

An especially interesting though controversial consequence of this rationale is that the human species, as being the latest emerging of big animals, is situated at the highest level of complexity. And in addition we may make a still more interesting observation, namely that there is an empty gap between our presently existing nearest relatives and us, because all intermediate pre-human species are extinct. This gap makes it easier to consider the human species as unique. I have discussed this issue at length in Ekstig (2016).

These features of evolution are illustrated by means of a diagram showing complexity versus time. I have in previous publications displayed and analysed this diagram being reproduced in Figure 1.

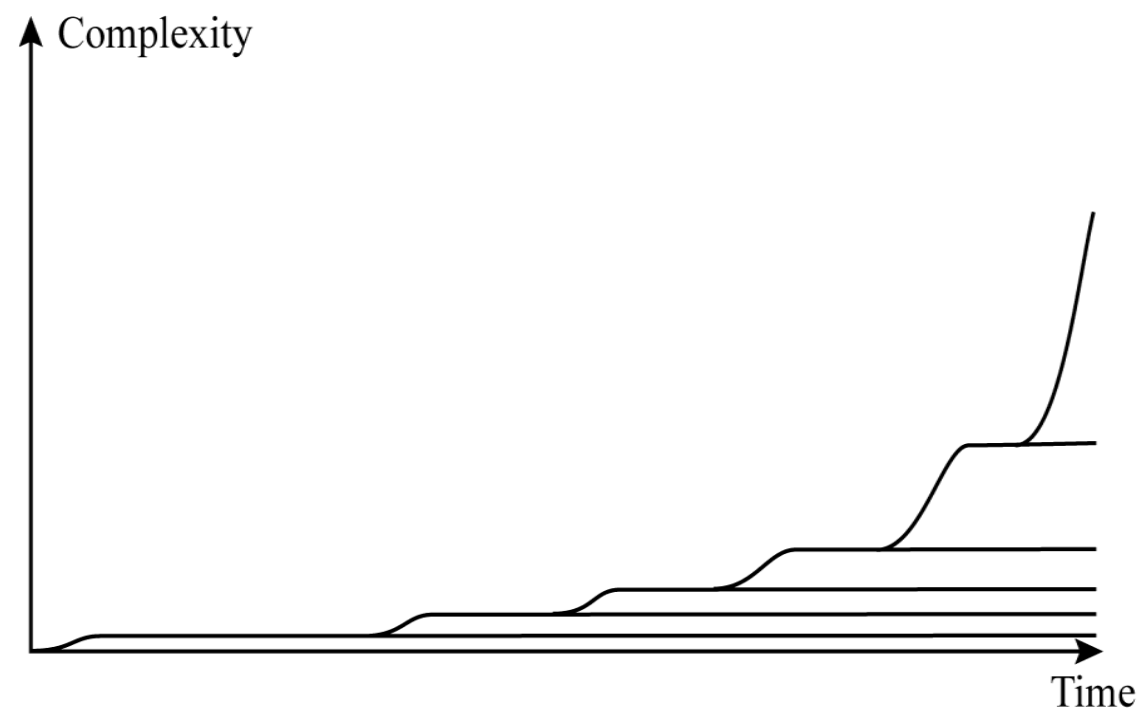

Figure 1. A diagram of evolution as formed by processes suggested in the present analysis. The diagram was first published in (Ekstig 2010 B) and repeatedly reprinted and discussed in (Ekstig, 2015, 2016).

The diagram of Figure 1 displays in a highly simplified form complexity versus time and can be interpreted to comprise organic as well as cultural evolution. The diagram can be seen as a Tree of Life. The horizontal lines represent stagnant species and the stepwise curve represents the emergence of novel species, a process that mainly occurs amongst those species that dwells at the highest level of complexity. This stepwise curve at the same time represents the common descent of all species and, maybe one could say, of all cultures as well. As a consequence of this view, one may understand why evolution proceeds cumulatively in a decisive direction in the complexity space towards ever-increasing complexity.

The curves in the diagram are explained as result of the action of competition as I have discussed at length in a previous work (Ekstig, 2015). The concept of competition is found to be applicable in cultural evolution as well. Thus Richard Alexander (1989) proposes that social competition between and within human groups has implied a feedback of intelligence constantly producing successively enhanced human intelligence.

Dawkins expresses a similar notion: "If a meme is to dominate the attention of a human brain, it must do so at the expense of 'rival' memes" (Dawkins, 1976 p. 211). Daniel Dennett articulates an analogous view: "Minds are in limited supply, and each mind has a limited capacity for memes, and hence there is a considerable competition among memes for entry into as many minds as possible. The competition is a major selective force in the infosphere and, just as in the biosphere, the challenge has been met with great ingenuity." (Dennett, 1995 p. 349). It is easy to observe that, for instance, there is an incessant competition between different religions and sects, which may be seen as a competition of space in people's brains. 
A much more detailed presentation of vast diversity of animal evolution is to be found in Dawkins' comprehensive survey (Dawkins, 2004), the principle difference of which in comparison with the present view is that it doesn't comprise the concept of complexity.

The steps in the uppermost curve in Figure 1 indicate especially rapid raises of complexity in connection with the emergence of novel species. Such changes are accomplished by natural selection, which, as I suggest, is supported and enhanced by other evolutionary mechanisms such as feedback, sexual selection, and arms race. These mechanisms are now discussed.

\section{Feedback}

The developmental growth of a living creature is governed by their inherited genetic set up. These genes are sieved by natural selection before fed back to the start of the development of the individuals of the next generation. Thus, as I previously have discussed (Ekstig, 2016), the transmission of genetic information from the adults to their offspring may be regarded as a feedback mechanism that is successively repeated in an iterative process all along the evolutionary course. Cultural knowledge, manners and values are in an analogous way transmitted to children during their upbringing, thus forming a similar process of feedback.

The principle of feedback is applied in electronic amplification devices, a mechanism that can give a much stronger (or weaker) amplification than that given by the amplifier itself. It works by means of a coupling of the output signal back to the input side thus forming a closed loop for the signal that in this way is repeatedly amplified. This mechanism is here suggested to have its analogy in the evolutionary process as well, inasmuch as genetic information, after being sieved by natural selection, is coupled back to the beginning of each new generation, thus forming a self-reinforcing lope of genetic information. This principle is relevant to cultural and social evolution as well. Thus, cultural characteristics in every society are fed back to the growing children in this way accomplishing continuing cultural qualities in human societies.

Bernard Crespi (2004) discusses the application of feedback to evolution. He points out that positive feedback can be instrumental in driving many of the most important and spectacular processes in evolutionary ecology. He emphasizes that the self-reinforcing dynamics of positive feedback generates the conditions for changes that might otherwise be difficult or impossible for selection or other mechanisms to achieve and that it can generate large-scale changes in genetic systems. Amongst examples of feedback, Crespi mentions the peacock's tail and the human brain.

I suggest the principle of feedback to be regarded as an inherent and inescapable principle of evolutionary change because, as a most fundamental feature of evolution, living creatures go through their individual developmental course with an onset at every new generation by just retaining and reusing the genetic and cultural information transmitted from the preceding generation. In this way an iterative loop of information is formed that may be successively changed during the developmental course - changes being statistically expressed in processes on the population level, the features of which may be successively displaced due to the influence of natural selection.

Take as an example the thickness of fur being a response to cold climate. In a climate of decreasing temperature, the fur grows until its function satisfactorily balances the benefit in relation to its cost. This is normal natural selection. In this process, the selection causes a change during the developmental course that might be compared to the amplification in the electronic device. After this sieving process, the changed genetic information is fed back from the adult to its offspring in a process causing evolutionary change over time. In the example of the fur, there will in this way be a cumulative growth of the fur. Needless to say, the fur exerts no impact on the climate and this is the common meaning of the mechanism of feedback. In this way feedback gives a complementary mechanism to natural selection. Most of evolutionary changes in all species can be explained as a result of these dual processes, leading to the continuous increase of complexity over the evolutionary course.

A manifestation of feedback can be seen in cultural evolution as well. Social habits and values, vernacular language, religious propensities, as well as general knowledge of how to live and survive are transmitted to the children. When grown-ups, they repeat this process thus forming a feedback process that in the course of human history has given rise to cultural and social stability but also to successive changes. An illustrative example is seen in religious activity.

Dawkins (2006 p. 176 - 177) points out that natural selection builds child brains with a tendency to believe whatever their parents and tribal elders tell them, because such a trusting obedience is valuable for survival. But a child has no way of distinguishing good advice from bad. When the child grows up and has children of her own, she will pass the whole lot on to her own children. Dawkins furthermore points out that religious leaders are well aware of the vulnerability of the child brain and the importance of getting the indoctrination in early. As I see it, one may regard this process as an example of ordinary feedback because children have no chance to argue against or have influence on their parents or priests. In most cases, this case of feedback is characterized by an intentional resistance towards change, a 
process leading to a quite conservative society. In discussing possible analogies between cultural and organic evolution one could compare the intentional resistance towards change with stabilizing selection.

\section{Strong feedback}

However, I think that feedback may have an additional, much more efficient form inasmuch as the result of the selection process may have an influence on the very environment by which the selection procedure is accomplished. I suggest this form of feedback to be called strong feedback. In returning to the analogy with electronic amplification devices, I think that one can regard strong feedback as a process in which the output of the amplification is influencing the core amplification of the amplifier, thus making the amplification still stronger. In the context of evolution the selection process accomplished by the environment is considered as corresponding to the core amplification. Strong feedback is an active recursive process that can be considered as a complementary mechanism to the passive adaptation to the environment characterizing natural selection. I give some examples of this mechanism.

\subsection{Sexual Selection}

A commonly discussed example of sexual selection is the conspicuous plumage of the peacock's tail. This feature in the males is reinforced by sexual preference for such traits in the females. The preferences in the females are inherited in forthcoming generations of females and the genes of females having the strongest expression of their preferences will be promoted by their mixing with the males with the most extravagant tails. Therefore, the preference in the females is also reinforced in the course of evolution. This mutual evolutionary process is discussed at length by Dawkins, pointing out that the genes for male qualities, and the genes for making females prefer those qualities are evolving together:

Every time a male is chosen because of his long tail, not only are genes for long tails being chosen. At the same time, because of the 'togetherness' coupling, genes for preferring long tails are also being chosen. What this means is that genes that make females choose males tails of a particular length are, in effect, choosing copies of themselves. This is the essential ingredient of a self-reinforcing process: it has its own self-sustaining momentum. Evolution having started in a particular direction, this can, in itself, tend to make it persist in the same direction (Dawkins, 1988 p. 206).

In the context of the present discussion of feedback, this mechanism is strong feedback, because the selection process is affecting the very environment by which it is accomplished, in this way promoting the participants' own reproduction a mechanism leading to the runaway evolutionary change since long being observed as a result of sexual selection.

Of course, natural selection due to the external environment may be in action as well, being simultaneously and independently superimposed to the achievements of sexual selection. It seems that sexual selection primarily gives the most conspicuous changes such as great plumage, ornamental colours and antlers. The application of sexual selection in the field of cultural evolution is discussed in sections 5.3 and 5.4.

\subsection{Arms Race}

The normal action of natural selection implies that the environment is unaffected by its impact on the creatures in the process. Arms race, in contrast, means a sharp deviation from this principle in that the selection process accomplishes an impact on the environment in which it is exerted. Therefore, according to my definition, this process is to be regarded as strong feedback. As Dawkins (2004 p. 496) points out, arms races are deeply and inescapably progressive in a way that, for example, evolutionary accommodation to weather is not.

A typical example is the impact predators and prey exerts on one other. Let as look at such an example. It is obvious that there is a strong selection pressure on gazelles to develop an ability to run fast in order to escape cheetahs, in this context considered as their environment. In this way the gazelles are compelled to run faster which implies a selection pressure on the cheetahs to run faster in their turn. This reasoning can be carried out in the other way too. I think that such an increasing ability implies a growth of complexity in the anatomy of both the involved species that may go on in the same direction until physical or physiological limitations set in. Thus I conclude that arms race can contribute to the steps of increasing complexity illustrated in Figure 1.

Arms race is in action in the human cultural and social evolution as well. Blackmore discusses arms race in the field of social evolution, in referring to the 'Machiavellian Intelligence' hypothesis. She thus states:

The similarity with Niccolò Machiavelli (1469 - 1527), the devious adviser of sixteenth-century Italian princes, is that much of social life is a question of outwitting others, plotting and scheming, entering into alliances and breaking them again. All this requires a lot of brain power to remember who is who, and who has done what to whom, as well as to think up ever more crafty wiles, and to double bluff the crafty wiles of your rivals - leading to a spiralling arms race (Blackmore, 1999 p.74).

This process of arms race in the social evolution of mankind has no doubt contributed to the rapid growth of our 
enormous brains and our intelligence. But maybe it isn't altogether beneficial because in the field of social evolution, the process of arms race has its most literal meaning. It is a regrettable characteristic of human history that there has been such a great activity of warfare. Individuals, tribes, counties and nations have been engaged in numerous conflicts, thus having incessantly initiated inventions of successively more effective weapons and strategic knowledge. Like its application in the biological field, social arms race forms a self-reinforcing strong feedback because each side will compel the other to take part in the race for better weapons. As we as rational beings know all too well, this process may end in a human apocalypse. My hope is that science, in fostering democratic thinking, may show a possible way to break this terrible spell.

\subsection{The evolution of verbal language}

The emergence of verbal language reasonably implied a great survival advantage for the early tribes of the human species, thus being rapidly evolved as a result of natural selection. However, there are another mechanisms acting in this process that have an additional influence, maybe even greater, on the evolution of language. Those mechanisms are imitation and sexual selection.

Blackmore suggests that imitation and sexual selection have important influences on the development of verbal language in human children. She maintains that people preferentially mate with and imitate people with the best language (Blackmore, 1999 p. 104). This means that children of parents with exceptionally good verbal faculties are brought up in a social environment having a stimulating effect on their language acquisition. When these individuals become parents to the next generation of children, the process is repeated. In this way, the social environment of a child is formed by the developmental process of the children in the preceding generation. Thus she concludes that sexual selection forms a crucial mechanism in the evolution of verbal ability in the human species.

However, one may raise the objection that parents with low verbal ability will have a slowing impact on their children, thus accomplishing the opposite effect on the evolution of language in the form of negative feedback process. This may be true, but I think that children in the small tribes of our ancestors certainly took part in common activities as for instance by sitting around the campfire and listening to the storytelling adults. In these situations, the most verbally talented adult person certainly dominated the talk and in this way the children were not solely dependent on their parents. In our own time there is a similar effect accomplished by schools in which children meet trained teachers that supposedly have a positive effect on their verbal development in addition to that of their parents.

In this way, children will enhance their verbal acquisition that, when they grow up, has a positive effect on the social environment for the next generation of children. This process thus means a self-reinforcing strong feedback mechanism.

I think this process, acting in addition to normal natural selection, is independent of the external physical environment because good language ability is certainly equally advantageous whether the physical environment is beneficent or deleterious. Verbal ability makes the brain visible in the process of sexual selection because, as Blackmore (ibid. p. 105) points out, being highly articulate makes you sexually attractive.

There is actually even a more direct coupling between the growing child and its social environment. Children have an inherent propensity of imitating other persons, especially their parents, and this is certainly of great significance for the acquisition of language. Indeed, it is observed that parents are stimulated to enhance the babies' inherited tendencies of imitation. Thus, as Patricia Kuhl (1915) describes, mothers are stimulated by their babies to use "baby talk", also denoted motherese, in their interaction with the babies, a form of language characterized by a high pitch, slow tempo and exaggerated intonation. She refers to research showing that motherese exerts a positive effect upon infant language learning.

Alison Gopnik and coauthors (Gopnik et al., 1999) point out that people across all cultures use motherese when they talk to their infants, even though they usually aren't aware of doing it at all. (ibid. p. 129) The authors speculate that it is the appearance of the babies' faces that initiate this behaviour and that this biological preference for baby faces seems to be instilled by nature. The authors thus maintain that there is coordination between what babies look like and what grown-ups think is cute. In this way grown-ups provide an environment in which babies can flourish (ibid., p. 166).

Thus it seems that in this way babies exert an influence upon adults. The babies, by means of their cute faces, initiate a reaction on the side of adults to use the motherese way of talking. This process, I think, has been evolved in the prehistoric evolution of our species in a mutually self-reinforcing process already from the very onset of language capability. In this sense, there is not only an impact from the adult to the child but from the child to the adult as well. The growing child thus accomplishes a change in its own environment that is of crucial importance for its early language acquisition. I thus conclude that language evolution is enhanced by a self-reinforcing strong feedback process.

I suggest that we may regard language evolution in the early human species as a transition period that goes from normal natural selection promoting improved survival to the form of self-sustaining evolution of memes that evolve, as Richard 
Dawkins points out, because they are advantageous to themselves (Dawkins, 1976 p. 214). The evolution of language thus seems to be enhanced by several cooperating processes: by means of ordinary natural selection due to its survival value, by a general feedback mechanism driven by imitation, by strong feedback driven by mutual imitation, and by sexual selection driven by the sexual attractiveness of language ability.

Because of the fact that the acquisition of language is by far the most demanding intellectual activity, this process has instigated the remarkable rapid growth of the human brain, a process that has advanced in spite of its great nutritional cost. I conclude that the growth of our intelligence, of course coupled to our big brain, is to be attributed to the activity of several cooperating mechanisms of which strong feedback provides an important factor.

\subsection{Memetic Selection}

The concept of memes was created by Dawkins (1976) as a parallel to genes, though applied to cultural evolution. As I suggest in the previous section, memes provide a mechanism for strong feedback in language evolution inasmuch as they exert influence on the environment in which they are selected. In a frequently cited formulation, Daniel Dennett points out that "the haven of all memes depend on reaching is the human mind, but a human mind is itself an artefact created when memes restructure a human brain in order to make it a better habitat for memes. .... The memes enhance each other's opportunities: the meme for education, for instance, is a meme that reinforces the very process of meme implantation." (Dennett, 1995, p. 365). As we can see, Dennett here articulates a rationale being exactly what I here have called strong feedback.

Due to this strong feedback process, memes cause more rapid evolutionary changes as compared to the week feedback in ordinary natural selection, in which there is no influence on the environment. This, I think, explains the common observation according to which culture is evolving much faster that organic evolution.

I have in previous works (Ekstig, 2010 A, 2015) suggested a method by means of which the rate of evolution is measured by the application of the concept of complexity. The result of this assessment implies that cultural evolution, occurring during the last million years, has contributed to complexity equally mush as organic evolution during the preceding billion of years. In the context of feedback, this result concurs with the present contention that strong feedback in its manifestations in cultural evolution can accomplish a much faster evolutionary change than what is typical for organic evolution.

\subsection{The evolution of science}

Science is a vital cultural activity in industrialized as well as developing societies. One may speculate in what way its evolution can be explained by similar processes as those of biological evolution.

The 1905 Swedish Nobel laureate in chemistry, Svante Arrhenius (1859-1927) expressed in a book from 1907 the notion of an analogy of scientific evolution to Darwinian evolution, thus at the time when the discussion of Darwin's ideas was intense:

It is with ideas as with organisms. A lot of seeds are sown, but only a few will grow, and amongst the living things being evolved from them, most are weeded out due to the struggle for existence, and only a few will remain living. In a similar way, ideas that are most successfully corresponding to nature are gradually selected. (Arrhenius, 1907. $p$. $175-176$, my translation from Swedish).

As we can see, Arrhenius associates the selection of ideas to the Darwinian concept of struggle for existence, in other words, to natural selection.

I have in my previous works as well as in the present emphasized the importance of the developmental process for the progress of organic evolution and for the human cultural and social progress. The connection between children's mental growth and the evolution of science was first observed by Jean Piaget by means of the concept of genetic epistemology. By means of this concept, Piaget suggested that there is a parallelism between the progress made in the logical and rational organization of knowledge found in the history of science and the corresponding formative psychological processes developed in children (Piaget, 1970 p.13).

I think, though, that there is more to this than a mere parallelism. It is generally contended that children's acquisition of knowledge during childhood and schooling has great significance for the progress of science. The results of scientific research are then transmitted back to school and university curricula, thus forming a self-reinforcing feedback process. However, there is a feedback coupling between the education of school children and the work of teachers as well, because the result of the training of teachers is dependent on the previous school education. This coupling means strong feedback. Although this process can be highly beneficial for the growth of welfare in a society it may unfortunately give rise to a vicious circle as well.

As an example, I refer to the present situation in Sweden, where there is a great shortage of students aspiring for 
training to a profession as teachers of mathematics and the natural sciences. This means a shortage of good teachers in these school subjects that might lead to a situation in which many pupils develop distaste for these subjects resulting in a still greater shortage of teacher students in these subjects in the future. In this case, then, we may talk about a negative feedback coupling and it seems important that school authorities are aware of such tendencies.

In their historical overview of the evolution of science, Holton and Brush (1973 p. 196) have convincingly pointed out the crucial importance of science for the promotion of progressive societies. They found it to be a source of constant wonder that once science got its first impetus in the early seventeenth century, it swept over Europe like a long pent-up storm. And when the Copernican and Newtonian - and the Darwinian, I would like to add - worldview triumphed in the West, the recognition that uniform laws hold sway over all matter - and life, I would like to add - everywhere helped overcome hierarchical thinking and prepare the minds for self-reliant democracy.

\section{Conclusion}

It is generally acknowledged that organic life has been and is subject of continuous change being generally seen as a result of adaptation to the prevailing environment. However, in considering the chaotic nature of the environment in which the selection is carried out, this process cannot in itself bring about a decisive direction to evolution. In order to identify such a direction one needs a measure of evolution and I have in previous publications suggested complexity to provide such a measure. This measure indicates that the evolutionary process is characterized by a more or less continuous increase of complexity.

The question of whether natural selection can provide an explanation for the human cultural evolution has long been subject to intensive discussion. In the present work I suggest some additional mechanisms for this process, actually being common to the organic evolutionary process as well. These mechanisms are competition and feedback.

The mechanism of competition in organic evolution can be associated to the popular expression "the struggle of life". In many instances, though, the competition from adjacent species is such that a species may find it best to stay at its level in the complexity space thus applying stabilizing selection. It is only species at the highest level of complexity that may evolve to a higher level because at these levels there are no competing species. This process is disruptive selection. On the basis of this reasoning I have constructed a new form of a Tree of Life in the form of a diagram of complexity versus time.

In the cultural form of evolution competition has likewise a high explanatory power. There is a limited room in the human brain bringing about competition of ideas, cultural expressions and norms.

The mechanism of feedback is explained by the fact that evolution proceeds in an iterative way, transmitting information from the adults to their offspring, thus under the ubiquitous pressure of competition giving selection the opportunity to repeatedly work on the developmental process and in this way steadily increase the amount of complexity. This reasoning is valid for cultural evolution as well.

I have in the present thesis suggested a new version of feedback called strong feedback. Strong feedback is suggested to be an active recursive selection process, characterized by an impact on the environment in which the selection is operating. This selection then implies a bearing on genes and memes encouraging their own reproduction thus causing an especially rapid evolutionary change in a specific, seemingly arbitrary direction. In this way strong feedback may explain features of evolution that Darwinian passive adaptation to the environment is incapable of doing.

An example is found in sexual selection in which there is a mutual coupling between the selection and its result. Sexual selection explains many anatomical traits and is in action in the evolution of verbal language as well inasmuch as there is a mutual coupling between the child and the adult.

Another example of strong feedback is found in arms race. Gazelles and cheetahs form each other's environment that gives rise to typical arms race. In the human society there may be individuals that attempt to deceit and wile one another, and to double bluff the crafty wiles of rivals, leading to spiralling arms race.

Other applications of strong feedback are found in the evolution of verbal language, in the process of memetic selection, and in the evolution of science.

The mechanisms of competition and feedback are suggested to be responsible to a large extent for the large-scale increasing measure of complexity in the evolutionary process. These mechanisms can explain the progressive nature of the evolutionary process, indeed as I venture to say, they explain the evolutionary trend towards ever higher heights. In addition, these mechanisms are not only active in the organic but in cultural evolution as well. I conclude that these mechanisms can be seen as complementary to the process of Darwinian natural selection inasmuch as they can explain features of the evolutionary processes that natural selection in its common interpretation is unable to do. 


\section{References}

Alexander, R. D. (1989). Evolution of the human psyche. In P. Millar \& C. Stringer (Eds.). The human revolution: Behavioral and biological perspectives on the origins of modern humans, 455-513. Princeton: Princeton University Press.

Arrhenius, S. (1907). Människan inför världsgåtan. Hugo Gebers. Stockholm.

Blackmore, S. (1999). The Meme Machine. Oxford: Oxford Univ. Press.

Blum, H. F. (1968). Time's Arrow and Evolution. Princeton: Princeton University Press.

Crespi, B. (2004). Vicious circles: positive feedback in major evolutionary and ecological transitions. Trends in Ecology and Evolution, 19(12).

Dawkins, R. (1976). The Selfish Gene. Oxford: Oxford Univ. Press.

Dawkins. R. (1988). The Blind Watchmaker. Penguin Books, London.

Dawkins, R. (2004). The Ancestor's Tale. Weidenfeld \& Nicolson.

Dawkins, R. (2006). The God Delusion. Bantam Press. London.

Dennett, D. C. (1995). Darwin's Dangerous Idea. New York: Simon \& Schuster.

Ekstig, B. (2010 A). Complexity and evolution: A study of the growth of complexity in organic and cultural evolution. Foundations of Science, 15, 263-278. http://dx.doi.org/10.1007/s10699-010-9179-6

Ekstig, B. (2010 B). Biological and Cultural Evolution in a Common Universal Trend of Increasing Complexity. World Futures 66, 435-448. http://dx.doi.org/10.1080/02604027.2010.489490

Ekstig, B. (2015). Complexity, Natural Selection and the Evolution of Life and Humans. Foundations of Science, 20, 175-187. http://dx.doi.org/10.1007/s10699-014-9358-y

Ekstig, B. (2016). Processes in Organic and Cultural Evolution. In Richardson, J. 2016. Natural Selection and Genetic Drift. Nova Science Publishers, Inc. New York.

Gopnik, A., Meltzoff, A. N., \& Kuhl, P. K. (1999). The Scientist in the Crib. William Morrow and Company, INC. New York.

Holton, G., \& Brush, S. (1973). Introduction to Concepts and Theories in Physical Science. Princeton University Press. Princeton.

Kuhl, P. K. (2015). Baby Talk. Scientific American. November 2015, 313, 64-69.

Lineweaver, C. H., Davies, P., \& Ruse, M. (2013). (Eds.) Complexity and the Arrow of Time. Cambridge: Cambridge Univ. Press.

Piaget, J. (1970). Understanding Causality. W. W. Norton \& Co. New York.

Wilson, E. O. (1998). Consilience. Alfred A. Knopf, New York.

\section{(cc) EY}

This work is licensed under a Creative Commons Attribution 3.0 License. 